(с) А.Г. Демина', В.Б. Бреговский', И.А. Карпова' ${ }^{1}$, Т.Л. Цветкова²

'Санкт-Петербургский территориальный диабетологический центр, Санкт-Петербург

²ОО «Новел СПб», Санкт-Петербург

ОБОСНОВАНИЕ. Неактивная стадия диабетической нейроостеоартропатии (ДНОАП) характеризуется отсутствием асептического воспаления и наличием необратимых деформаций стопы. Однако в настоящее время остается неясным, изменяются ли форма пораженной стопы и ее биомеханика в отдаленные сроки наблюдения.

ЦЕЛЬ. Изучить изменения распределения нагрузки под пораженной стопой у больных с неактивной стадией ДНОАП.

МЕтоДы. Обработаны результаты повторных компьютерных педографий (платформа emed AT, novel GmbH) у 27 больных (8 мужчин и 19 женщин) с односторонней неактивной ДНОАП. Пиковое давление (ПД) рассчитывалось в процентах к исходному и отдельно для периодов наблюдения: до 24 мес, 24-48 мес, более 48 мес.

РЕзУЛЬтАТЫ. Прирост ПД в конце наблюдения: под большим пальцем на 50\%, под 1-м плюснефаланговым суставом (ПФС) - на 30,7\%, под 2-м пальцем - на 20\%, под 2-м ПФС - на 6\%, под средним отделом - на 9\%. Под 3, 4, 5-м пальцами ПД снизилось на 67\%. Достоверные изменения в первые 24 мес наблюдения отмечены только под 3, 4, 5-м пальцами: ПД уменьшилось на 62\%, далее только на 9\%. Увеличение нагрузки в других областях появилось в первые 24 мес, однако достоверным стало при сроках наблюдения до 48 мес. Максимальных значений показатели ПД в сравнении с исходными достигли в сроки более 48 мес. Наибольшее увеличение нагрузки выявлено под большим и 2-м пальцами, 1, 2, 3-й плюсневыми головками, средним отделом.

ЗАКЛЮЧЕНИЕ. В неактивную стадию ДНОАП происходит постепенное перераспределение нагрузки под стопой, что указывает на изменение ее формы. Давление увеличивается под большим и 2-м пальцами, 1-3 ПФС, средним отделом и снижается под 3-5-м пальцами. Эти изменения нарастают со временем, становясь наиболее выраженными через 4 и более лет наблюдения. Полученные данные могут быть полезны при дифференцированном обеспечении больных с неактивной ДНОАП сложной ортопедической обувью.

КЛЮЧЕВЫЕ СЛОВА: сахарный диабет; диабетическая остеоартропатия; стопа Шарко; физиология походки; компьютерная педография

\title{
CHANGES IN LOADING DISTRIBUTION IN PATIENTS WITH CHARCOT FOOT DURING LONG-TERM FOLLOW-UP
}

\author{
(c) Anastasia G. Demina', Vadim B. Bregovskiy' ${ }^{1}$ Irina A. Karpova', Tatiana L. Tcvetkova²
}

'Saint-Petersburg City Diabetes Centre, St.-Petersburg, Russia

${ }^{2}$ LLC Novel SPb, St.-Petersburg, Russia

BACKGROUND. The inactive stage of the diabetic Charcot arthropathy foot (CA) is characterised by fixed foot deformities and an absence of inflammation. However, it remains unclear if the shape of the foot and its biomechanics change during long-term follow-up.

AIM. To evaluate changes in loading distribution of the affected foot, in patients with inactive CA, during long-term follow-up.

MATERIALS AND METHODS. Twenty seven patients with unilateral inactive CA (19 females, 8 males) were studied. Computer pedography (emed AT, novel gmbh) was performed and baseline and the last studies were analysed. Maximal peak pressures (PP) were obtained for the first and the last studies and the percentage of the PP change was calculated for the total follow-up period and for periods: $<24$ months, $24-48$ months, $>48$ months.

RESULTS. PP increased: under the hallux 50\%; 1st metatarsal-30.7\%; 2nd toe-20\%; 2nd toe-6\%; midfoot-9\%. PP decreased under $3-5$ toes up to $67 \%$. Significant changes at the first period were found under $3-5$ toes only $(-62 \%)$. The increase in loading under the other parts of the foot appeared at 24 months; however, these changes became significant between 24 and 48 months and peaked after 48 months of follow-up. The maximal increase of PP was noticed under the hallux, the 2nd toe, metatarsals $1-3$ and the midfoot. 
CONCLUSIONS. We revealed the gradual redistribution of PP, under the different parts of the foot, in patients with inactive CA. This redistribution reflects changes in the shape of the affected foot. The loading increased under the hallux, the 2 nd toe and the corresponding metatarsals, 3rd metatarsal and midfoot, and decreased under the 3-5 toes. These changes increased during the follow-up, becoming more pronounced after 4 or more years. Our data may be useful for constructing custom-made footwear for patients with CA.

KEYWORDS: diabetes mellitus; diabetic osteoarthropathy; Charcot foot; gait physiology; computer pedography

Неактивная стадия диабетической нейроостеоартропатии (ДНОАП) характеризуется отсутствием асептического воспаления и наличием необратимых деформаций стопы [1, 2]. Поэтому предполагается, что в неактивную стадию изменений формы стопы, ассоциированных с течением артропатического процесса, уже не происходит. Из этого предположения следует вывод об относительной стабильности формы пораженной стопы во времени при отсутствии гнойно-некротических осложнений ДНОАП и оперативного лечения.

Однако клинический опыт и данные отдельных исследований заставляют усомниться в справедливости подобных рассуждений. Так, состояние наружного и внутреннего сводов пораженных стоп в сравнении с контралатеральной стопой было изучено при помощи рентгенографии под нагрузкой через 6, 12 и 24 мес после первичного установления диагноза активной ДНОАП [3]. Если выявленное авторами прогрессирующее опускание наружного и внутреннего сводов, а также разрушение кубовидной кости в течение 6 и 12 мес с момента установления диагноза ДНОАП представляется очевидным и обусловлено, скорее всего, активностью артропатии, то прогрессирование изменений сводов через 24 мес, когда ДНОАП, вероятно, перешла в клинически неактивную стадию, с активным артропатическим процессом связать нельзя. Следовательно, можно полагать, что в неактивную стадию форма пораженной стопы меняется, и соответственно этим изменениям перераспределяется нагрузка под стопой, аналогично тому, как это происходит с больными, перенесшими малые ампутации [4]. В свою очередь, знание закономерностей перераспределения нагрузки под пораженной стопой при диспансерном наблюдении пациентов с ДНОАП может повлиять на тактику профилактических мероприятий и, прежде всего, обеспечение индивидуальными ортопедическими изделиями.

\section{ЦЕЛЬ}

Изучить изменения распределения нагрузки под пораженной стопой у больных с неактивной стадией ДНОАП.

\section{МЕТОДЫ}

\section{Дизайн исследования}

Исследование носило ретроспективный характер. Проводилось сравнение результатов компьютерной педографии, повторно выполнявшейся у пациентов с неактивной ДНОАП в рамках диспансерного наблюдения перед очередным заказом сложной ортопедической обуви. Для этого из 170 больных с неактивной ДНОАП, которым выполнялась педография, были отобраны 27 больных с повторными исследованиями, которые удовлетворяли критериям соответствия. Сравнивали результаты первого и последнего из повторных исследований за период с 1998 по 2017 гг.

\section{Критерии соответствия}

Отбор данных компьютерной педографии для последующего анализа производился при наличии следующих условий:

1. Клинически и рентгенологически неактивная односторонняя ДНОАП среднего отдела в течение более 12 мес.

2. Наличие минимум двух педографий с промежутком между ними более 12 мес.

3. Отсутствие малых ампутаций на стопе с ДНОАП и операций, нарушающих форму этой стопы в период между первой и последней педографиями (например, вскрытий флегмон, остеотомий или тенотомий).

4. Отсутствие на момент обследования язвенного дефекта стопы на стопе интереса или на контралатеральной стопе.

5. Исследование выполнено при ходьбе без дополнительной опоры (трость, костыли) и без посторонней помощи.

6. Пациент не применял на момент исследования ортопедический аппарат или тутор.

7. Отсутствие постуральной неустойчивости, сенсомоторной атаксии, пареза нижней конечности любой этиологии, снижения остроты зрения и сужения его поля как состояний, препятствующих педографии на платформе.

\section{Условия проведения}

Исследование проведено на базе амбулаторного кабинета «Диабетическая стопа» Санкт-Петербургского Территориального диабетологического центра в составе Санкт-Петербургского ГБУЗ «Городской консультативно-диагностический центр №1».

\section{Продолжительность исследования}

Медиана времени между первым и повторным исследованиями составила 36 мес, при этом минимальный промежуток между первым и последним исследованием составил 19 мес, максимальный - 192 мес.

\section{Описание медицинского вмешательства}

Оценка распределения давления под стопами в динамике (при перекате) проводилась при помощи компьютерной педографии. Для измерения плантарного давления применялась платформа emed-a50 (novel gmbh, Германия). Использовался протокол первого шага с 5 измерениями для каждой стопы и последующим усреднением результатов. При наличии нескольких измерений в анализ отбирались данные первого и последнего обследований. 
Таблица 1. Характеристика групп в зависимости от времени между первым и вторым обследованием

\begin{tabular}{lccc}
\hline \multicolumn{1}{c}{ Параметр } & $\mathbf{1 8 - 2 4} \mathbf{~ M e c ~}$ & $\mathbf{2 4 - 4 8}$ мec & >48 \\
\hline Число пациентов & 10 & 8 & 9 \\
Возраст, лет* & $49,4 \pm 12,6$ & $52,3 \pm 16,2$ & $53,4 \pm 13,7$ \\
Длительность сахарного диабета, лет* & $20,2 \pm 17,3$ & $19,6 \pm 13,9$ & $26,1 \pm 12,8$ \\
Мужчины/женщины & $2 / 8$ & $3 / 5$ & $3 / 6$ \\
і8і9Тип сахарного диабета (1/8іu2) & $4 / 6$ & $4 / 4$ & $7 / 2$ \\
\hline
\end{tabular}

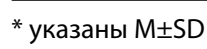

\section{Основной исход исследования}

Основным измеряемым при педографии показателем исследования являлось пиковое давление (ПД). Величина ПД рассчитывалась отдельно для следующих областей стопы: большой палец, 1-й плюснефаланговый сустав (ПФС), 2-й палец и 2-й ПФС, 3-5-й пальцы, 3, 4 и 5-й ПФС, средний отдел стопы и пятка. Так как целью исследования являлась оценка динамики ПД, в качестве расчетного параметра был принят процент прироста или уменьшения ПД при сравнении исходного и повторного измерения.

\section{Дополнительные исходы исследования}

В качестве дополнительных исходов исследования выбрано изменение ПД в подгруппах в зависимости от величины временного промежутка между первым и последним исследованием.

\section{Анализ в подгруппах}

В связи с тем, что частота заказа новой ортопедической обуви существенно варьировалась у разных пациентов (от ежегодного заказа до 1 пары в 2-3 года), временной промежуток между первой и повторной педографиями значительно колебался. Поэтому расчет показателей нагрузки производился для следующих периодов между первым и вторым измерением: от 18 до 24 мес, от 24 до 48 мес и более 48 мес. Соответственно этим временным промежуткам обследованные разделились на три группы (табл. 1).

Пациенты всех трех групп значимо не отличались по возрасту и продолжительности сахарного диабета.

\section{Методы регистрации исходов}

Регистрация и анализ ПД производились при помощи программного обеспечения novel $\mathrm{GmbH}$, основанного на математическом анализе сигнала датчиков, встроенных в диагностическую платформу. Для хранения измерений использовалась база данных novel-database diabetes.

\section{Этическая экспертиза}

Исследование одобрено Этическим комитетом при ФГБУ «НМИЦ им. В. А. Алмазова» Минздрава России (протокол №22 от 11.02.2013). Все пациенты подписывали информированное согласие на лечебно-диагностические мероприятия в рамках врачебного приема в Санкт-ПетербургскогоГБУЗ «Городской консультативно-диагностический центр №1».

Статистический анализ

Размер выборки предварительно не рассчитывался. Данные представлены как среднее или медиана. Срав- нение давления в разные временные периоды проводилось при помощи теста Манна-Уитни. Различия считались достоверными при уровне значимости менее 0,05. Статистический анализ проведен при помощи Statistica 6.0. for Windows.

\section{РЕЗУЛЬТАТЬ}

\section{Объекты (участники) исследования}

В анализ были включены данные педографии 27 больных, исходная характеристика которых приведена в табл. 2.

Пациенты характеризовались длительным анамнезом заболевания, а минимальный период времени, прошедший после верификации неактивной стадии, составил 18 мес.

Основные результаты исследования

На рис. 1 отражены изменения ПД при последнем измерении в процентах к исходной его величине.

Отмечено достоверное увеличение нагрузки на большой палец и 1-й ПФС. При этом прирост ПД под большим пальцем был значительно больше, чем увеличение ПД под 1-м ПФС: медиана прироста $50 \%$ (от $-66 \%$ до $826 \% ; p=0,0005$ ) и $30,7 \%$ (от $-51 \%$

Таблица 2. Характеристика пациентов при первом обследовании

\begin{tabular}{lc}
\hline \multicolumn{1}{c}{ Параметр } & Величина \\
\hline Возраст, лет* & $51,6 \pm 10,3$ \\
Длительность сахарного диабета, лет* & $22,7 \pm 12,5$ \\
Мужчины/женщины & $8 / 19$ \\
Тип сахарного диабета (1/2) & $15 / 12$
\end{tabular}

Длительность наблюдения после

завершения активной стадии, медиана 36 (18-192)

(мин-макс)

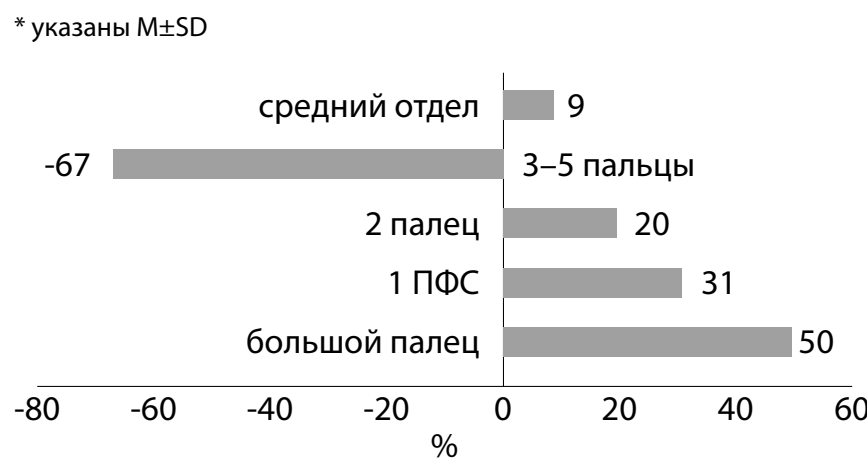

Рис. 1. Динамика пикового давления исходно и при последнем обследовании, \% к исходной величине. 
до $157,2 \% ; p=0,003)$ соответственно. Также произошло значительное увеличение ПД под 2-м пальцем: на 20\% в сравнении с исходным (от -87,7 до 436\%; $p=0,0001$ ), при этом отмечалась недостоверная тенденция к увеличению ПД под 2-м ПФС (+6\%; от -50 до +167\%). Выявлено уменьшение участия 3, 4, 5-го пальцев в процессе переката, которое выражалось в снижении величины ПД под ними: медиана снижения давления колебалась от 23 до 100\% и составила 67\% (p=0,001). Напротив, нагрузка под средним отделом несколько возросла $(p=0,05)$.

\section{ДОПОЛНИТЕЛЬНЫЕ ИСХОДЫ ИССЛЕДОВАНИЯ}

С учетом выявленных тенденций мы проанализировали изменения характера распределения плантарного давления в зависимости от времени наблюдения. Характеристика больных в зависимости от времени, прошедшего между первым и вторым обследованием, представлена в табл. 1 (см. выше). Данные измерений представлены на рис. 2.

В первый временной период (от 18 до 24 мес) отмечена тенденция к увеличению ПД под большим пальцем и областью первого ПФС. Напротив, ПД под 3-5 пальцами в этот период значительно уменьшилось $(p=0,001)$, но при дальнейшем наблюдении достоверного изменения давления под этими пальцами не произошло. В остальных областях стопы в сроки до 24 мес ПД также увеличилось, но эти изменения не достигли уровня достоверности.

В период от 24 до 48 мес изменения под большим пальцем и 1-м ПФС стали значимыми $(p=0,01)$. В то же время на $18 \%$ увеличилась нагрузка на 2-й ПФС $(p=0,04)$, однако величина давления под другими ПФС значимо не изменилась. Примечательно, что в сроки до 48 мес нагрузка под средним отделом и 2-м пальцем значимо не изменилась.

На сроках наблюдения более 48 мес происходило значимое увеличение нагрузки под 1-м и 2-м лучами (1-й и 2-й палец и соответствующие им плюсневые кости), 3-м ПФС, а также под средним отделом. При этом наибольший прирост ПД отмечен под большим пальцем (в 11 раз в сравнении с исходным). Динамика давления под 4, 5-м ПФС была недостоверна.

\section{Нежелательные явления}

Во время исследований нежелательных явлений не отмечено.

\section{ОБСУЖДЕНИЕ}

Резюме основного результата исследования

У больных с большими сроками неактивной стадии ДНОАП при помощи компьютерной педографии нами было выявлено значительное увеличение ПД под большим пальцем и 1-м ПФС и в меньшей степени под 2-м пальцем и средним отделом и уменьшение ПД под 3-5-м пальцами. Эти изменения указывают на прогрессирование деформаций в неактивную стадию.

\section{Обсуждение основного результата исследования}

В опубликованных ранее работах вопросы биомеханики стопы Шарко изучались в контексте особенностей распределения нагрузки и определения порогового значения для развития язвенных дефектов стоп. Основное внимание исследователей привлекал средний отдел стопы, так как известно, что поражения этого отдела наиболее опасны с точки зрения развития язвенно-некротических осложнений $[5,6]$.

В результате проведенного нами исследования впервые получены данные о характере перераспределения нагрузки под пораженной стопой у больных с неактивной ДНОАП в течение длительного периода наблюдения. Изменения формы стопы с ДНОАП в динамике на срокахдо 24 мес были зафиксированы рентгенологически Hastings M. et al. [3]. Однако, так как эти авторы не пользовались МРТ-критериями или сцинтиграфией для констатации завершения активной стадии, то нельзя быть уверенным в том, что они оценивали неактивную стадию. Более того, в настоящее время хорошо известно, что МРТ-критерии (исчезновение отека костного мозга) перехода ДНО АП в неактивную стадию могут запаздывать в сравнении с клинико-рентгенологическими критериями на 2-3 мес [7].

Принимая во внимание эти сведения, мы оценивали только тех больных, сроки наблюдения у которых после клинического завершения активной стадии превышали 12 мес (минимальный срок после окончания активной стадии составил 18 мес). Кроме того, клиническим критерием перехода в неактивную стадию в нашей работе была температурная разница менее $1^{\circ} \mathrm{C}$, а не $2^{\circ} \mathrm{C}[8,9]$. Это позволило нам предположительно свести к минимуму возможность того, что в группу попадут больные с остаточной активностью ДНОАП.

Так как изменение нагрузки на определенные отделы стопы невозможно без изменения ее формы, то в нашем исследовании при применении функциональной методики впервые получены сведения о прогрессировании деформаций в неактивную стадию 1. Выявлены следующие тенденции.

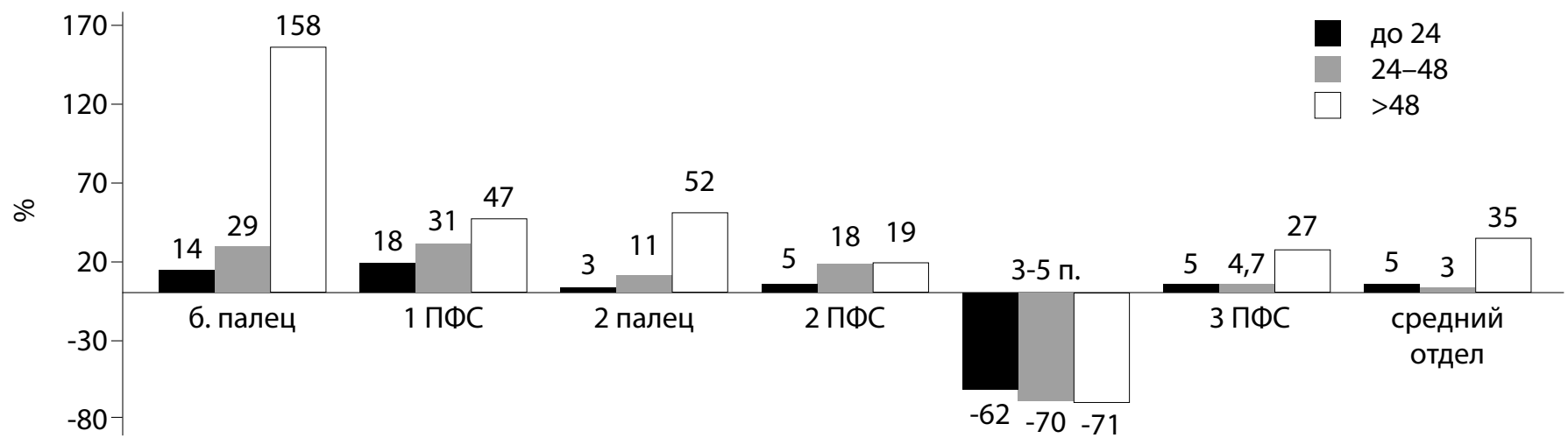

Рис. 2. Изменение пикового давления под стопой с ДНОАП в процессе наблюдения, \% к исходной величине 
1. Быстрее всего происходит тыльная девиация 3-5 пальцев и уменьшение участия их в заднем толчке, что, с одной стороны, проявляется снижением нагрузки под ними, с другой - увеличением вероятности травмы тыла крючковидных пальцев об обувь. При этом ПД в области 3, 4, 5-го ПФС в первые 4 года существенно не нарастает, но затем увеличивается только под 3-м ПФС, по-видимому, вследствие опускания головки 3-й плюсневой кости в плантарную сторону и атрофии подкожно-жирового слоя. Возможно, также играет роль и ее центральное расположение при перекате стопы с поражением среднего отдела, и, в особенности, при валикообразной деформации.

2. Выявленное нами увеличение нагрузки на средний отдел, наиболее вероятно, обусловлено опусканием сводов стопы, которое обнаружили Hastings M. et al. [3]. Нами показано, что эти изменения происходят только в отдаленные сроки наблюдения (более 4 лет), и прирост ПД относительно невелик в сравнении с большим или 2-м пальцами. Тем не менее, отмечается прогрессирование «валикообразности» стопы.

3. Значительное усиление нагрузки на 1-й луч объясняется нарастанием ригидности 1-го ПФС и формированием молоткообразной деформации большого пальца. Эти изменения появляются достаточно быстро и через 2 года уже статистически значимы.

4. Аналогичная закономерность справедлива и для 2-го луча: формирование крючковидного 2-го пальца и ригидного 2-го ПФС, при этом нагрузка под 2-м пальцем нарастает быстрее, чем под ПФС, что указывает на меньшее участие 2-го ПФС в опоре и толчке, в сравнении с 3-м ПФС, где нагрузка выше. Наиболее вероятно, что это обусловлено теми же процессами, которые отмечены в п.1.

Природа изменений формы стопы, вызвавших выявленную нами динамику распределения нагрузки под стопой с течением времени, до конца не изучена. С одной стороны, патология связочного аппарата, обусловленная неферментативным гликированием и изменением соотношения коллаген/эластин, изменения мягких тканей, характерные для синдрома диабетической стопы, атрофия мышц стопы могут приводить к прогрессированию деформации стопы $[10,11]$. С другой стороны, нельзя исключить и влияния ослабления связочного аппарата и атрофии мышц голени вследствие длительной иммобилизации на этапе лечения активной стадии.

С практической точки зрения полученные результаты имеют значение для изготовления сложной ортопедической обуви. Известно, что возможность выполнения педографии в практическом здравоохранении отсутствует. Поэтому, не проводя исследование и опираясь на выявленные закономерности, можно построить план обеспечения обувью в зависимости от времени, прошедшего от перехода в неактивную стадию. В частности, исходя из динамики нагрузки, при ортопедическом снабжении в первые 2 года следует уделять повышенное внимание глубине обуви и качеству ее верха в области пальцев. В более поздние сроки, кроме этого, необходимо предпринимать меры к уменьшению нагрузки на область большого и 2-го пальцев, 1, 2, 3-го ПФС, а также к увеличению загрузки пятки и уменьшению нагрузки в области среднего отдела при наличии валикообраз- ной деформации. При больших сроках к предыдущим мерам добавляется ряд конструктивных особенностей обуви, позволяющих еще в большей степени компенсировать нарастающие биомеханические аномалии. Наши данные также показывают, что в первые два года после установления неактивной стадии ДНОАП допустимо изготавливать сложную обувь по одной колодке, а стельку - по одному слепку. В последующие годы необходимо либо корректировать предыдущую колодку, либо изготавливать ее заново, и, соответственно, использовать старый слепок для стельки также нельзя. С другой стороны, при диспансерных осмотрах (не реже 1 раз в квартал) нужно ориентировать больных в отношении повышенного внимания к областям высокого риска развития язв пораженной стопы с учетом временной последовательности увеличения нагрузки на них.

\section{Ограничения исследования}

На конечный результат и выявленные тенденции могло оказать влияние ограничение чувствительности использованной нами педографической платформы, верхний предел измерения которой составляет 1275 кПа. Подобная нагрузка, в частности, была зарегистрирована у 5 пациентов исходно и при повторном обследовании в области среднего отдела и у одного больного под большим пальцем, поэтому при подсчете динамики это отражалось как отсутствие изменений. Таким образом - повтор с учетом небольшой выборки, мы можем оценивать только тенденцию, однако можно предполагать, что при повышении верхнего порога измерения платформы усредненные цифры относительного прироста нагрузки под средним отделом были бы существенно больше, что соответствует нашему клиническому впечатлению при наблюдении за этими пациентами. Все измеряемые параметры у больных с ДНОАП, как правило, характеризуются высокой вариабельностью вследствие сложной формы стопы, многообразия деформаций, постуральной неустойчивости. Нам представляется, что относительно небольшой, в сравнении с другими областями стопы, прирост ПД под средним отделом обусловлен именно этими факторами, которые играют наибольшую роль в фазе полной опоры при перекате.

\section{ЗАКЛЮЧЕНИЕ}

В неактивную стадию ДНОАП происходит постепенное перераспределение нагрузки под стопой, что указывает на изменение ее формы. Давление увеличивается под большим и 2-м пальцами, 1-3-м ПФС, средним отделом и снижается под 3-5-м пальцами. Эти изменения нарастают со временем, становясь наиболее выраженными через 4 и более лет наблюдения. Полученные данные могут быть полезны при дифференцированном обеспечении больных с неактивной ДНОАП сложной ортопедической обувью.

\section{ДОПОЛНИТЕЛЬНАЯ ИНФОРМАЦИЯ}

Источник финансирования. Исследование не имело целевого финансирования. Сбор данных выполнялся в рамках лечебно-диагностического процесса, а их статистическая обработка - во внерабочее время. 
Конфликт интересов. Авторы декларируют отсутствие явных и потенциальных конфликтов интересов, связанных с публикацией настоящей статьи.

Участие авторов. Демина А.Г. - клиническое обследование больных, организация и ведение базы данных, написание статьи; Брегов- ский В.Б. - разработка общей концепции исследования, проведение компьютерной педографии, участие в написании статьи; Карпова И.А. редактирование текста статьи; Цветкова Т.Л. - статистическая обработка данных, полученных в результате педографии, редактирование статьи.

\section{СПИСОК ЛИТЕРАТУРЫ | REFERENCES}

1. Rogers LC, Frykberg RG, Armstrong DG, et al. The Charcot foot in diabetes. Diabetes Care. 2011;34(9):2123-2129. doi: 10.2337/dc11-0844

2. Milne TE, Rogers JR, Kinnear EM, et al. Developing an evidence-based clinical pathway for the assessment, diagnosis and management of acute Charcot Neuro-Arthropathy: a systematic review. J Foot Ankle Res. 2013;6(1):30. doi: 10.1186/1757-1146-6-30

3. Hastings MK, Johnson JE, Strube MJ, et al. Progression of foot deformity in Charcot neuropathic osteoarthropathy. J Bone Joint Surg Am. 2013;95(13):1206-1213. doi: 10.2106/JBJS.L.00250

4. Bregovskiy $\vee$, Tsvetkova T, Demina A. Biomechanical changes in patients with diabetes during follow up. In: Proceedings of the 11 th Scientific Meeting of the Diabetic Foot Study Group; 2013 Sep 20-22; Sitges, Spain

5. Armstrong DG, Peters EJG, Athanasiou KA, Lavery LA. Is there a critical level of plantar foot pressure to identify patients at risk for neuropathic foot ulceration? J Foot Ankle Surg. 1998;37(4):303-307. doi: 10.1016/s1067-2516(98)80066-5

6. Armstrong DG, Lavery LA. Elevated Peak Plantar Pressures in Patients Who Have Charcot Arthropathy. J Bone Joint Surg. 1998;80(3):365-369. doi: 10.2106/00004623-199803000-00009
7. Zampa V, Bargellini I, Rizzo L, et al. Role of dynamic MRI in the follow-up of acute Charcot foot in patients with diabetes mellitus. Skeletal Radiol. 2011;40(8):991-999. doi: 10.1007/s00256-010-1092-0

8. Armstrong DG, Lavery LA. Acute Charcot's Arthropathy of the Foot and Ankle. Phys Ther. 1998;78(1):74-80. doi: 10.1093/ptj/78.1.74

9. Демина А.Г., Бреговский В.Б., Карпова И.А. Критерии продолжительности иммобилизации пораженной конечности при диабетической нейроостеоартропатии Шарко // Сахарный duaбem. - 2014. - T. 17. - №4. - C. 60-65. [Demina AG, Bregovskiy VB, Karpova IA. Criteria of immobilization duration of the affected foot in diabetic Charcot neuro-osteoarthropathy. Diabetes Mellitus. 2014;17(4):60-65. (In Russ.)] doi: 10.14341/dm2014460-65

10. Bus SA, Maas M, Cavanagh PR, et al. Plantar Fat-Pad Displacement in Neuropathic Diabetic Patients With Toe Deformity: A magnetic resonance imaging study. Diabetes Care. 2004;27(10):2376-2381. doi: $10.2337 /$ diacare.27.10.2376

11. Bus SA, Maas M, Michels RP, Levi M. Role of intrinsic muscle atrophy in the etiology of claw toe deformity in diabetic neuropathy may not be as straightforward as widely believed. Diabetes Care. 2009;32(6):1063-1067. doi: 10.2337/dc08-2174

\section{ИНФОРМАЦИЯ ОБ АВТОРАХ [AUTHORS INFO]}

Бреговский Вадим Борисович, А.М.н. [Vadim B. Bregovskiy, MD, PhD]; адрес: 194354 СанктПетербург, ул. Сикейроса, д. 10Д [address: 10D Sikeyros str., St-Petersburg, 194354 Russian Fedration]; ORCID: http://orcid.org/0000-0002-5285-8303; eLibrary SPIN: 2547-3330; e-mail: podiatr@inbox.ru

Демина Анастасия Геннадьевна, врач-эндокринолог [Anastasia G. Demina, MD]; ORCID: http://orcid.org/0000-0001-8126-8452; eLibrary SPIN: 6161-8594; e-mail: ans.dem@bk.ru Карпова Ирина Альбертовна, К.М.н. [Irina A. Karpova, MD, PhD]; ORCID: http://orcid.org/0000-0002-2390-8404; eLibrary SPIN: 7691-6058; e-mail: iakar@mail.ru

Цветкова Татьяна Лазаревна, к.т.н. [Tatiana L. Tcvetkova, PhD technical sciences]; ORCID: http://orcid.org/0000-0001-6684-0422; eLibrary SPIN: 7360-9173; e-mail: tatianatsvetkova@inbox.ru

\section{ЦИТИРОВАТЬ:}

Демина А.Г., Бреговский В.Б., Карпова И.А., Цветкова Т.Л. Изменения распределения нагрузки под стопой Шарко в отдаленные сроки неактивной стадии // Сахарный диабет. - 2018. - Т. 21. - №2. - С. 99-104. doi: 10.14341/DM9388

\section{TO CITE THIS ARTICLE:}

Demina AG, Bregovskiy VB, Karpova IA, Tcvetkova TL. Changes in loading distribution in patients with Charcot foot during long-term follow-up. Diabetes Mellitus. 2018;21(2):99-104. doi: 10.14341/DM9388 